\title{
Research on Concanavalin A Crystallization in a Flow-Free Droplet-Based Device
}

\author{
Guan-ru DU ${ }^{1}$, Wen-jun ZHANG ${ }^{1,2}$, Ding-kun $\mathrm{JI}^{3}$, Feng-juan $\mathrm{CHEN}^{1}$, \\ Di YIN ${ }^{1}$, Hong-bo ZHANG ${ }^{1}$, Rui-xue YIN $^{1}$, Xiao-peng $\mathrm{HE}^{3}$ \\ and Shih-Mo YANG ${ }^{1, a,{ }^{*}}$
}

${ }^{1}$ Complex and Intelligent Research Center, School of Mechanical and Power Engineering, East China University of Science and Technology, Shanghai, PR China

${ }^{2}$ Department of Mechanical Engineering, University of Saskatchewan, Saskatoon, SK, Canada

${ }^{3}$ Key Laboratory for Advanced Materials and Institute of Fine Chemicals, School of Chemistry and Molecular Engineering, East China University of Science and Technology, Shanghai, PR China

asmyang@ecust.edu.cn

${ }^{*}$ Corresponding author

Keywords: Protein, Crystallization, Droplet-based.

\begin{abstract}
Protein crystallography is a very critical process that limits the development of pharmaceutical engineering. Microfluidic technology has provided an opportunity for protein crystallization. In this paper, a flow-free droplet-based device is presented for producing highly controlled crystals and fabrication of this device with polydimethylsiloxane (PDMS) is presented. The experiment was performed on the device and shows that the crystals of Con A and protein ligand (DK3) have a spherical structure.
\end{abstract}

\section{Introduction}

Although in the past few decades, the research of protein crystallography has made prominent progress, it still remains to a very critical process which limits the development of pharmaceutical engineering [1]. The three-dimensional structure information on protein allows a structure-based drug design for development of cost-effective and highly efficiency drugs [2]. X-ray crystallography is the method frequently used to determine the structure of proteins which provides key biological insights to the three-dimensional structure and function of proteins and provides the essential information on protein-protein and protein-ligand interactions [3]. However, X-ray sources do not provide enough capacity to produce detectable diff raction for a single protein molecule, also the success of X-ray crystallographic studies much depends on the quality of the crystals [4]. The way to obtain protein crystals is still a difficult problem for the structural determination of proteins due to its complexity and difficulties [5].

In the recent years, microfluidic technology has turned to a feasible platform for nano-volume protein crystallization [6]. Several liquid-handling robots are developed to ease the manpower and time-consuming screening task and can accurately dispense volumes as small as $50 \mathrm{~nL}$ [7]. Nevertheless, their high cost and complexity make them inaccessible to the vast majority of researchers [8]. So far there are three major types of microfluidic devices dedicated to protein crystallization: (a) valve-based systems,(b) 
droplet-based systems, and (c) SlipChip systems [9]. In the droplet-based system crystallization takes place inside aqueous volumes surrounded by an immiscible carrier fluid and the flow-free droplet-based chip leads a useful method for crystallization research [10].

This paper reports an experiment based on the flow-free droplet-based method to generate the protein crystals of Con A and protein ligand (DK3).

\section{Experimental Section}

\section{Materials and Chemicals}

Concanavalin A (Con A) was purchased from Sigma-Aldrich, USA, stored at $-20{ }^{\circ} \mathrm{C}$ to retain the activity of protein. The DCM-grafted DK3 solution was acquired from Key Laboratory for Advanced Materials and Institute of Fine Chemicals of ECUST, store at $-20{ }^{\circ} \mathrm{C}$ to avoid water absorption. $\mathrm{NaOH}$ and dimethyl sulfoxide, DMSO (>99\%) was purchased from Lingyun Chemical Technology. Deionized water $(\mathrm{PH} \approx 7.1)$ was extracted from the purification system from Ulupure Purification Technology. PDMS (Sylgard 184 elastomer base) and its curing agent (Sylgard 184 elastomer kit) were purchased from Dow Corning, USA. Mineral oil also purchased from Dow Corning, USA. PP Tube (3ml) was purchased from Zhizhong Biotech, China. The magic tape was purchased from $3 \mathrm{M}$ Scotch, USA.

\section{Microfluidic Chip Fabrication}

Photolithography is one of the vital microfabrication technique which utilizes transferring microscale patterns to photosensitive materials by means of selective exposure to optical radiation [11].

A standard lithographic procedure was followed to the fabrication of the mold for crystallization chip. A silicon wafer with a spin-coated flat uniform film was consisted by a photosensitive material. The micro scale of micropillars array device was designed by using computer-assisted design software such as AutoCAD 2000 (Autodesk, USA) in this research. Then the pattern was transferred into a specific format for photomask printing (CAD/Eastcom Lingtong Electronics Enterprise Co., China). The photomask used as the standard photolithography when exposure of SU-8 photoresist to high-level UV light defined the pattern of the design by preserving some area of the photoresist from UV onto the silicon wafer. A silicon substrate with micropillars was shaped after the UV exposed area become soluble in the developer solution of $1.0 \%(\mathrm{~m} / \mathrm{V}) \mathrm{NaOH}$ for 2.5 minutes then washed by deionized water and termed development in the drying oven at $120{ }^{\circ} \mathrm{C}$ for 20 minutes.

The elastomer base and its curing agent of PDMS were blended together with the proportion of 10:1 then the mixture was degassed in a vacuum chamber for 30 minutes to remove the trapped air bubbles. The crystallization chip was wrapped around by tape in order to make a pond where the mixture of PDMS was poured, followed by cured at $70{ }^{\circ} \mathrm{C}$ for 120 minutes. The microwell array was fabricated on the chip after the solidified PDMS structure was separated from the mold. The array contains 670 round cavities with the diameter of $200 \mu \mathrm{m}$ over $1 \times 1 \mathrm{~cm}^{2}$ area. The Lithographic procedure used to construct crystallization chip is shown in Fig 1. 


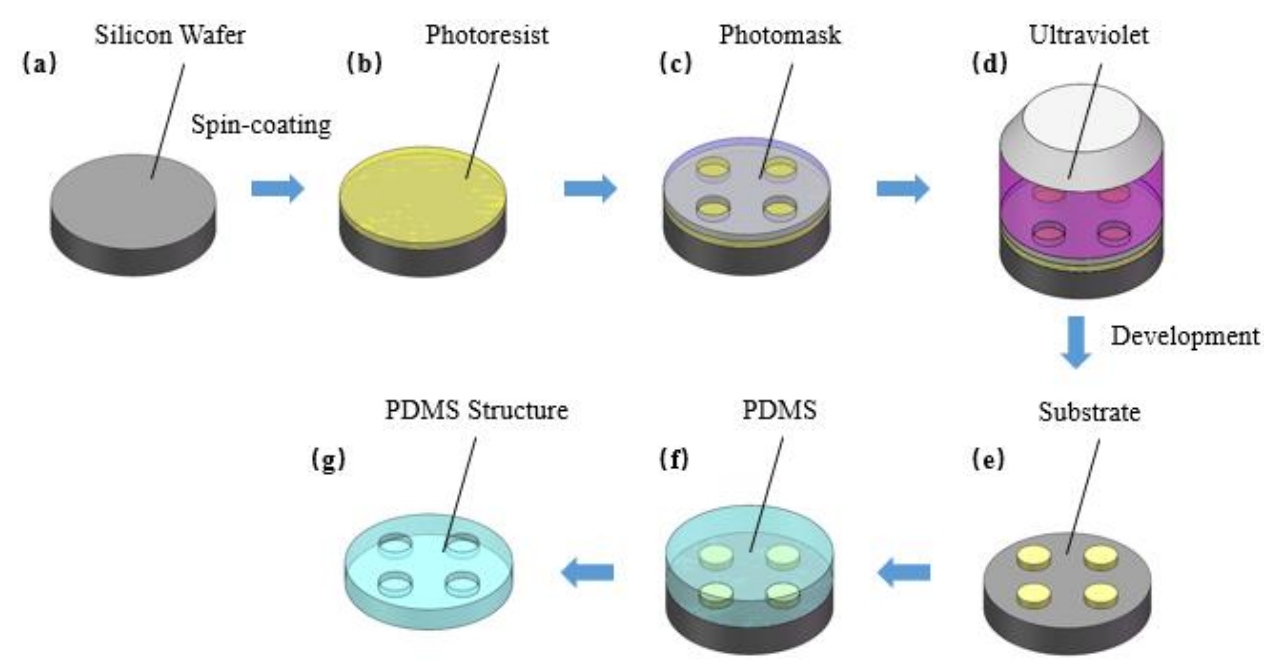

Figure 1. Lithographic procedure used to construct crystallization chip

\section{Droplet Package Procedure}

PDMS with the properties of low air permeability and solubility can absorb air from its surrounded circumstances when the air within is degassed. This droplet preparation technique takes advantage of this feature to make the crystallization chip in the following steps.

First, the crystallization chip was degassed previously in vacuum conditions for 40 minutes for evacuation of air dissolved in the PDMS. The chip surface was covered with liquid immediately after the chip was fetched out, followed by the air in the cavities gradually permeating into the PDMS bulk to decline the air pressure in the microwells. With the air volume reducing correspondingly, the liquid was driven into the microwells automatically by the discrepant gas pressure.

Next, the redundant liquid on the surface of the chip removed after all of the microwells were brimmed with the solution by the surface tension. Mineral oil was applied to cover the chip surface totally to separate the trapped solution from external air.

Finally, the water molecules inside the droplets dissolved into mineral environment little by little in normal condition leading to the volume of droplets reduce slowly which moved the solubility of droplet toward the supersaturation required for nucleation and crystal growth. After water dissolved entirely in the mineral oil, a single clear crystal was formed in each microwell.

\section{Methods}

In this paper, all experiment processes performed at $20{ }^{\circ} \mathrm{C}$ to ensure an identical and stable environment for crystallization. The Con A solution was prepared by dissolving $0.5 \mathrm{mg}$ Con A (lyophilized powder) in $1 \mathrm{ml}$ DMSO and was stored overnight at $4{ }^{\circ} \mathrm{C}$. DK3 solution was acquired at a concentration of $3 \mathrm{mg} / \mathrm{ml}$ followed by diluted to $0.03 \mathrm{mg} / \mathrm{ml}$ as a stock solution. The sample was prepared the Con A/DK3 mixture by the desired amount of the stock solution was subsequently transferred into tubes to mixing the Con A solution and to produce the final protein solution.

After $0.25 \mathrm{ml}$ protein solution was added to the degassed crystallization chip, the microwells were filled with the solution by the PDMS bulk absorbed the air from the cavities gradually in 5 minutes, and thus the liquid was poured in and this process was driven by the discrepant gas pressure automatically. Then remove the needless liquid on the surface of the chip and add mineral oil cover the chip surface immediately. 
The process of water molecules dissolving into mineral environment resulted in the volume of droplets reducing slowly. At this stage, the solubility of droplet moved toward the supersaturation required for nucleation and crystal growth. Crystallization was allowed to occur in $\sim 48$ hours. After water dissolved entirely in the mineral oil, the crystal was formed in each microwell.

\section{Result and Discussion}

Optical microscopy was conducted by a Nikon Eclipse Ti equipped with a digital camera. The growth and nucleation of protein with our droplet-based method mark a significant difference in individual crystal forming in the respective microwell. The final Con A with DK3 crystals, as shown in Fig 2(b) (c), were well-formed with spherical models influenced by the ligand-protein interaction.

The size distribution of the protein crystals was shown in Fig. 2(d). The length of the spherical structure was calculated as the size of the protein crystal. The mean size of protein crystals in $200 \mu \mathrm{m}$ diameter micro-wells was $53 \mu \mathrm{m}$, a standard deviation (sd) was 2.57 , so the coefficient of variance $(\mathrm{CV})$ was $4.85 \%$, less than $5 \%$, which indicated that crystal sizes can be precisely controlled in this study and this method can obtain uniform particles.

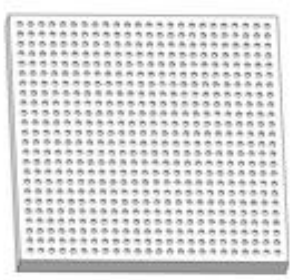

(a)

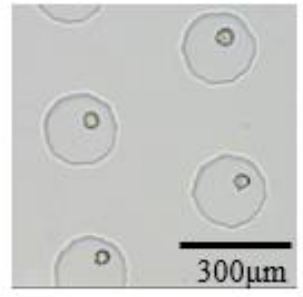

(b)

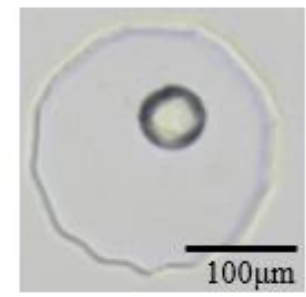

(c)

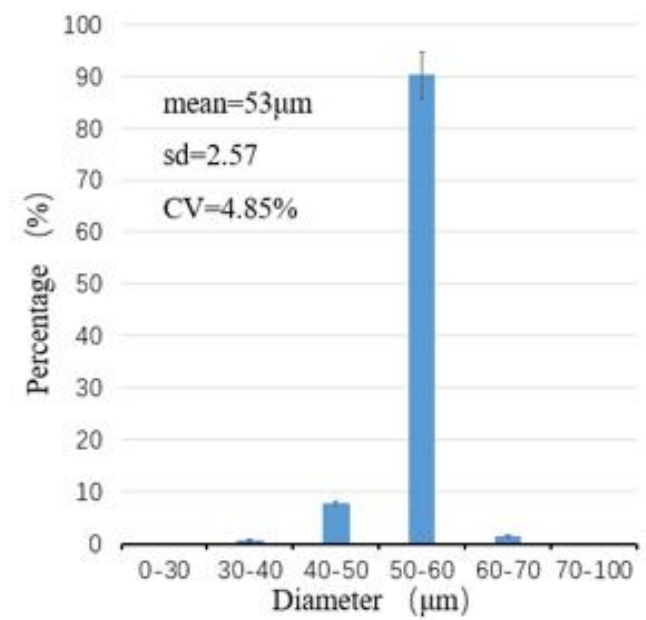

(d)

Figure 2. (a) The schematic of the flow-free droplet-based device (b) The spherical protein crystals in the micro-well array (c)The appearance of single spherical crystal (d) Size distribution of protein crystals

\section{Conclusion}

We demonstrated a droplet-based microfluidic device to crystallize protein. The device utilized microwells array to ensure the high-throughput and uniform crystals. The crystallization of Con A with DK3 indicated the new type of combination. Crystallographic analysis revealed that the framework of protein was facilitated by inducing ligand. Moreover, this device is convenient for studying and statistics of the crystal parameters 


\section{Acknowledgement}

This work was supported by Complex and Intelligent Research Center, School of Mechanical and Power Engineering, East China University of Science and Technology. The authors would like to thank the financial support of National Natural Science Foundation of China (21404038) and the Fundamental Research Funds for the Central Universities of China (22A201514029)

\section{References}

[1] Maeki, Masatoshi, et al., Microfluidic Approaches for Protein Crystal Structure Analysis, Analytical Sciences 32.1 (2016) 3-9.

[2] Cheng, Alan C., et al., Structure-based maximal affinity model predicts small-molecule druggability, Nature biotechnology 25.1 (2007) 71-75.

[3] McPherson, Alexander., Introduction to protein crystallization, Methods 34.3 (2004) 254-265.

[4] Abdallah, Bahige G., et al., Protein crystallization in an actuated microfluidic nanowell device, Crystal growth \& design 16.4 (2016) 2074.

[5] Liu, Yue, et al., A protein crystallisation screening kit designed using polyethylene glycol as major precipitant, CrystEngComm 17.29 (2015) 5488-5495.

[6] Lau, Billy TC, et al., A complete microfluidic screening platform for rational protein crystallization, Journal of the American Chemical Society 129.3 (2007) 454-455.

[7] Ouyang, P. R., et al., Overview of the development of a visual based automated bio-micromanipulation system, Mechatronics. 17 (2007) 578-588.

[8] Wang, Li, et al., A centrifugal microfluidic device for screening protein crystallization conditions by vapor diffusion, Sensors and Actuators B: Chemical 219 (2015) 105-111.

[9] Li, Liang, and Rustem F. Ismagilov, Protein crystallization using microfluidic technologies based on valves, droplets, and SlipChip, Annual review of biophysics 39 (2010) 139-158.

[10] Yang, Shih-Mo, et al, A flow-free droplet-based device for high throughput polymorphic crystallization, Lab on a Chip 15 (2015) 2680-2687.

[11]Huh, Dongeun, Geraldine A. Hamilton, and Donald E. Ingber., From 3D cell culture to organs-on-chips, Trends in cell biology 21.12 (2011) 745-754. 